\title{
Quality of life of patients with early onset dementia in Nigeria
}

\author{
Elias Olukorede Wahab*, Chioma Joan Ikebudu \\ Department of Sociology, Lagos State University, Lagos, Nigeria \\ *E-mail address: eliasphd@yahoo.com
}

\begin{abstract}
Dementia is a major cause of disability and mortality among the elderly, it becomes early onset of dementia, when it occurs before the age of 60 years. It is the loss of cognitive abilities, particularly the loss of memory; it appears to be one of the most dreaded conditions of old age. Around $5 \%$ of the population aged 65 and above is affected by dementia, and its prevalence rises with the rate nearly doubling every 5 years. The study used the key informant interview research tool to achieve its aim and objectives, thereby collecting data to answer its research questions. Purposive sampling was used, while ethnographic summary was used to analyse the data. The results of the study showed that the quality of patients with early onset of dementia is generally poor, in that the illness brings about the crippling of finance, loss of memory and independence, loss of position in the society. The study recommends detecting factors that can prevent or postpone the disease, and educating the public on early onset of dementia (OED). Interventions that could delay early onset of dementia would have a major positive public health impact and the prospect of dementia prevention may also reduce some of the common fears and anxieties of becoming older.
\end{abstract}

Keywords: Quality of Life; Patients; Dementia; Older Persons; Nigeria

\section{INTRODUCTION}

Old age is a feature in the population of the developing countries of the world. According to Mbanefo (2002), ageing of a population has grave consequence for the economic, socio-cultural, psychological and spiritual activities in all societies, especially in most developing countries, such as Nigeria. A major implication is that, the number of the economically active labour force will be reduced, which leads to a drastic reduction in Gross Domestic Product (GDP), as well as the percent income of the region.

Therefore, government and all national stakeholders have a significant responsibility to those vulnerable groups of the elderly, as old age is accompanied by an often preventable significantly diminished physical capacity, this suggests that the health of older people is deteriorating due to poverty, which continues to exist, except provision and adequate supervision is given to the elderly in Nigeria.

Old people are seen compensated with the fact that they are being taken care of by their adult children, grandchildren, spouses, siblings and even the extended family. To Aroun (2004:1): "Old age is revered, if for nothing else, but its gift of longetivity and wisdom. Because of their knowledge, the aged serves as advisers to their communities. They play a significant role in the extended family relations that is prevalent in Africa". In the words of 
Togonu-Bickersteth (2001), the current economic hardship with is accompanying high level of unemployment led many aged people in the rural areas to experience a condition called "de facto childlessness". Dementia is not a specific disease as it refers to a group of symptoms caused by brain disorders. It is general term used to describe the deterioration of mental activities. Memory loss is a common symptom of dementia, but it is not the only symptom. Other symptoms include losing the ability to solve problems, control your emotions and successfully communicate verbally.

Early onset Dementia (EOD), usually refers to someone who develops dementia regardless of the age it starts, even before the age of 65 changes in the brain can be caused by disease/trauma, and can result in loss of cognitive functioning, which includes memory, thinking, reasoning, decision making and verbal communication. It sometimes results in behavioral or personality changes, which all impedes on the quality of life of an individual, which includes consumer spending and production measures. Age is the greatest risk factor for dementia; the condition affects one in 14 people, over the age of 65 and rises steeply to one in six, over the age of 80.

Some patients respond to cognitive training, such as memory exercises. For patients, whose dementia, include behaviour problems, treatment with behaviour modification may result in some improvement. EOD can be difficult to diagnose, even when signs of early dementia are present. It has a profound effect on the quality of life of patients, as its symptoms are serious, the sense of loss for the people, with EOD are enormous which are the following:

- Memory problems, especially short term memory loss

- Poor judgment and language problems, which includes the pronouncement of words

- Erratic changes in moods, behaviour and personality.

- Disorientation in time, place and the person

- Difficulties in recognition, understanding and comprehension

- Agitation and Hallucination

The diagnosis given as early stage of dementia is a long process, as patient need to be monitored consistently, and tested overtime in order to confirm EOD (Pratt, 2009), as the condition can be confused with other mental health issues, such as depression, psychosis or brain tumour. According to Selmat (2008), "the heart of any research project, is the problem". Dementia is not merely the problem of memory, it reduces the ability to learn reason, retain or recall past experiences, and there is also a loss of pattern of thoughts, feelings and activities (Gelder et al 2005).

The complications that come with early onset dementia, has an impact on the quality of life (QOL) of patients. There is an interruption of family life, as patients may still be raising children; there is the loss of employment, and also the crippling of finances. There are losses in independence and initiative, leading to inability to take care of oneself and also nonparticipation in social activities, leading to social exclusion loneliness.

Burden of Early Onset Dementia (EOD) combined with possible loss of employment can cripple a family's financial situation. However, while the diagnostic process of early onset dementia. Is taking place, dementia symptoms can wreak havoc on personal relationships and work obligations. EOD places a significant burden on the individual, family and the immediate community. The implication of EOD on the QOL of patients is that the patient's behaviour and abilities get diminished and distorted. Therefore, What is early-onset dementia (EOD)? How can early-onset dementia (EOD) have an effect on the physical, socioeconomic, emotional well being of the patient? What causes early-onset dementia, can it 
bring about possible loss of employment, which brings forth financial handicaps? Does social status have anything to do with management of dementia? These and other questions shall be probed into in the course of this study.

\section{LITERATURE REVIEW AND THEORETICAL FRAMEWORK}

Dementia is a collection of symptoms including memory loss, personality change, and impaired intellectual functions resulting from disease or trauma to the brain. These changes are severe enough to impact daily living, independence, and relationships. With dementia, there will likely be noticeable decline in communication, learning, remembering, and problem solving. These changes may occur quickly or very slowly over time. The progression and outcome of dementia vary, but are largely determined by the type of dementia and which area of the brain is affected. Diagnosis is possible through advanced brain imaging, clinical examinations, and diagnostic testing. Dementia is early onset, in an individual when it occurs before the age of 60 or 65 years, (Cummings and Benson, 1992).

Some research suggests that people with early onset dementia decline at a faster rate. 1 in 10 cases of dementia in younger people may be treatable (proper diagnosis is essential but often not available in all cases). Patients may need genetic counseling and support. (Genetic defects on chromosomes of individuals are responsible for early onset dementia in a small number of families). Dementia, typically referred to as Alzheimer's disease (AD), and the vascular dementias $(\mathrm{VaD})$, represent the most common irreversible dementia syndromes (Cummings \& Benson, 1992). Less common dementias include dementia due to Parkinson's disease, Lewy body disease, Pick's disease, frontal-temporal dementia and progressive dementing disorders such as Huntington's disease and Creutzfeldt-Jakob disease. All dementias are characterized by impairment in multiple cognitive domains including memory, language, problem solving, judgment and abstraction, abilities, and skilled movement (Zec, 1993). Dementias also may be associated with psychiatric symptoms (e.g. hallucination, Delusions), behavioral disturbances, (e.g. agitation), personality changes (e.g, irritability), and disturbances of affect (e.g. depression, emotional liability) (Burns, 1992; 1996; Gilley, 1993). The main distinction between $\mathrm{AD}$ and $\mathrm{VaD}$ is disease course, with $\mathrm{AD}$ showing a pattern of steady progressive deterioration whereas $\mathrm{VaD}$ may show a more stepwise deterioration (Metter \& Wilson, 1993).

Although the type of cognitive and functional impairments experienced by demented individuals is well delineated, the demented individual's subjective experience of these impairments is not. The likely effect of limited information about the subjective experience of the disease is that treatable distress and excess disability may go undetected, remaining abilities and psychological resources underutilized, and means of coping and adaptation unaffiliated (Cohen, 1991; Cotrell \& Schulz,1993; Harrison, 1993; Kitwood, 1990; Kitwood, 1993; Kitwood \& Bredin, 1992).

What is known about demented individuals' subjective experience of the disease,comes from a mixture of clinical interviews and observation. For example, Solomon (1982) conducted one- to two-hour semi-structured interviews with 86 individuals diagnosed With Alzheimer's disease. Depressive symptoms were common, particularly in those individuals in the early stages of dementia who were more aware of their problems.

These symptoms usually reflected grief over loss of intellectual abilities and capacity to function independently, coupled with diminished feelings of mastery and control. In both early and middle stage individuals, panic and specific fears of becoming incapacitated and of 
passing on the disease were observed as well as frustration and anger directed at others. Suspiciousness was generally reported by those in the middle stage. Individuals appeared to cope with these stresses in different ways, including self-blame, somatization, blaming others, minimization of the severity of the impairment, and denial of the condition (by avoiding naming or gathering information about it).

\section{IMMUNOLOGICAL THEORY}

Defines ageing as a disease of the immune system, the immune system protects us from invading microorganisms and typical mutant cells that form within the body (i.e. cancer). Immune system acts in 2 ways: (1) by generating antibodies that react with the proteins of foreign organisms, (2) by forming cells that engulf and digest foreign cells. The production of antibodies and the ability to recognize mutant cells peaks during adolescence and decreases over time. The body loses its ability to distinguish between foreign agents/antigens and necessary body cells. The immune system becomes self-destructive and reacts against itself. Examples of autoimmune disease are lupus, scleroderma and adult-onset diabetes.

The immune system theory of aging is that the rate of aging is largely controlled by the immune system. As we age, the numbers of critical cells in the immune system decrease and become less functional. Starting before age 20, the thymus (which produced certain immune cells) begins to shrink. The immune system is the most important line of defense against foreign substances that enter the body. With age the system's ability to produce necessary antibodies that fight disease declines, as does its ability to distinguish between antibodies and proteins. In a sense the immune system becomes self-destructive and reacts against itself. Examples of autoimmune disease are lupus, scleroderma and adult-onset diabetes.

Decline in immune system function can negatively affect the outcomes of many illnesses such as pneumonia, urinary tract infections, postoperative infections, and cellulitis in the elderly adult. There is an age-associated decline in $\mathrm{T}$ cell functioning, which is accompanied by a decrease in resistance. The reduction in immunologic activities also leads to an increase in autoimmune dysfunction with age. Examples of autoimmune diseases are rheumatoid arthritis, lupus, and multiple sclerosis. A breakdown in the body's immunological memory system causes it to mistake normal cells as foreign and these are attacked by the person's own immune system.

Proponents of the immunological theory believe that a healthy diet and lifestyle coupled with preventive health measures, such as influenza and pneumonia vaccinations and limiting exposure to pathogens, can support immune function and help prevent ageing and untimely death. With the increase in number of auto antibodies, the number of attack also increases over the body tissues. Several diseases like Hashimoto's thyroiditis, atrophic gastritis are all involved to the ageing. These diseases occur after the initiation of the process of the body.

\section{DATA AND METHODS}

The study population concerns the population of Federal Neuro-psychiatric hospital, which includes the inmates, outpatients, the doctors and social workers of the hospital. The inmates, are also known as inpatients that stay in the hospital, for the purpose of treatment 
and are being cared for by the health givers (doctors, nurses, e.t.c.), the study population includes patients who are of the ages of 45-60 and above. The outpatients are patients of the hospital, who come on certain days, to see the doctor, for treatment, that is, for advice and medicine to make them stable, amongst them can be found the adults, the aged and also young adults of 18 and above. These patients can be involved in schooling or any employment position.

The sampling technique used is non-probabilistic, which is any sampling procedure, with no pre-assigned probability of including a particular unit into the sample. Purposive sampling is used in this study; sample size for this research work is 25 , which include the number of patients, doctors and social workers, who would be key informants.

The research instrument is key informant interview method (KII) to be used with the doctors and social workers of the patients in the hospital; it is a conversation between the interviewer and the respondent, with the purpose of eliciting certain information, this is possible through the following:

- Cognition or understanding by the respondent of what is required.

- The respondent has knowledge of the required information.

- Motivation on the part of the respondent to answer the questions accurately.

Data analysis involves the organization, rearrangement and presentation of data, in such a way, to the research questions. It also brings about the accurate identification, description and explanation of the nature of interrelationships in the study population. The method of data analysis is qualitative method of analysis. Ethnographic summary is to be utilised, Quotes would be identified, using exact words that are descriptive, representative of the respondents' views, and illustrate a particularly interesting perspective of one participant or respondent.

\section{RESULTS}

\section{1. Socio-demographic characteristics of respondents}

It was seen from information collected, more than a quarter of the respondents' claimed to be fifty-two years old. Half of the respondents were between the ages of sixty to sixty-five years, while six patients are sixty-five and above. This data imply that early onset dementia affects individuals who are old and it also affects the adults.

From data collected, it was discovered that the patients that were respondents, more than half were females, while a quarter were males; this implies that there are more females than males, who are patients or that care to come to the hospital. This implies that early onset of dementia has a link with sex, it attacks females than males.

From the findings, it was discovered that half of the respondents were married, with a quarter separated, and less than half patients were divorced, and another two who were widowed. Thus, this shows that patients with early onset of dementia in Federal Neuropsychiatric hospital, Yaba, are mostly married ones.

From the data collected, a quarter of the respondents, have Islam as a religion, also there were a quarter patients who were Christians, while two who were outpatients said they practiced traditional religion, and came to the hospital as a last resort. This shows that majority of early onset dementia patients either has Christianity or Islam, as a religion.

For analysis, a quarter of the respondents, who were both in and out patients, were Igbo, more than half of the respondents, hailed from the western part of Nigeria, with some patients, who 
says they hailed from Balyesa state. This finding indicates that most patients are from the western region of Nigeria.

The data collected revealed that, all of the respondents had a primary, and secondary education more, more than a quarter of the patients (both in and out) had a tertiary education. There were two outpatients, of which one is an outpatient and the other is originally an inpatient, which had beyond a B.Sc. This implies that early onset of dementia patients get education, when the condition does not have to do with memory.

Of the respondents, in which less than half were inpatients, they were not currently working, while the other respondents who were outpatients, were currently working, excluding two outpatients who said they were not working, as they were provided for by his offsprings. This shows that some patients can cope with the condition, when it does not affect their memory. Findings reveal that the respondents had several family types, less than half of the inpatients and outpatients, who were respondents, had polygamous families, others had monogamous families. The polygamous families had grown up children, who take on responsibilities, unlike the monogamous families had, in which the children were not yet grown up and are still dependent on their parents. This shows that families have a role to play in the quality of life of their family member who is a patient of early onset dementia.

\section{2. Causes of early onset of dementia}

A result of the study revealed that early onset of dementia is a loss of cognitive abilities which includes the ability to speak, think, and reason rationally. Some think it is just a loss of memory, which brings about irrational behavior. One of the key informants interviewed, who is a doctor, emphasizes that:

\section{"Dementia is a loss of brain function, which occurs with certain diseases. It affects memory, thinking, language and judgment".}

According to the respondents, they all claimed it had to do with the loss of memory, though there all several types of dementia, they all occur with the existence of Alzheimer's disease, which disrupts the areas of the brain that has to do with memory, movement, language, judgement, behavior and abstract thinking. A doctor, who was a key informant, had this to say:

\section{"Alzheimer's disease is the most common form of dementia, it accounts for two-thirds of all diagnosed cases, and medication can delay the onset of more debilitating symptoms".}

Results of the study also show that there is relativity in the existence of dementia, in an individual; it can be early, middle or late-onset. As there are some individuals, who are at the early stage of dementia. A doctor from the hospital said that:

"Early onset of dementia can also affect the adults who
are in their 30 s, $40 \mathrm{~s}$ or 50s. It usually first appears as
forgetfulness. This stage is called mild cognitive
impairment (MCI); it is the stage between normal
forgetfulness and the development of dementia".

A respondent who is a patient, but an out-patient responded saying: 
"As I am 52 years, but am having problems remembering something done yesterday, when I was working, this condition worried me, as I did not used to keep remember the appointments I kept and so I missed them. But now, the medication I take makes the condition not to interfere with my activities and I am aware of it, I also take memory exercises. The doctors say dementia might not occur, and call this condition of mine, mild cognitive impairment".

Findings also shows that majority of people with early onset dementia are between the age of 50-65, and above, and are either in or outpatients of Federal Neuro-psychiatric, hospital. Little is known about the prevalence of dementia, but Alzheimer's disease, which is the loss of memory, happens to be the most common, followed by vascular dementia. A doctor, said:

"Old age, body mass index of $55 \mathrm{~kg}$ or less and female gender are the significant risk factors".

Another doctor added by saying

"Family history of dementia and rural residence, below

the age of 25 can also be risk factors".

\section{3. Effects of early onset of dementia}

Result of the study shows that early onset of dementia impacts negatively on the physical, socio-economic, emotional wellbeing of patient. Respondents agree that the condition impacts negatively, in their life, as their social life disappears, there is emotional turmoil, and depression sets in, there is no more trust, the behavior is hard to handle, with regard to wandering and aggressive behavior. One of the respondents, a patient, supported by a social worker, said this:

"The illness really affects an individuals' life in the aspect of being healthy physically, one cannot move from place to place, while in some cases, the individual is fit like me, but emotional disturbances come into the illness."

This quote by this inpatient is quite different, as it is believed that dementia also affects thinking of a patient, but this was also supported by a social worker, who says:

"There are different patients here, who have different types of dementia, some affect memory, some affects movement, that results into strokes, some others affect nerve cells."

Loss of employment is also eminent, which brings forth financial handicap and a loss of status. This was corroborated by a doctor at the health institution, who says:

"In early onset of dementia, as it affects individuals with age, there is an increased likelihood that the patient was working and had to change or leave his or her job; even 
their caregivers might also have to leave a job in order to care for them, resulting in a double loss of income".

A social worker, in the hospital had this to say:

"The patients lose their memory, so they cannot work and are forced into early retirement and this affects their health, as some of them do not have enough money to buy the requirements needed for treatment".

Burden of Early onset dementia can cripple a family's financial situation, a social worker, who works closely with the patients, opined:

"All the time, these patients do not have money for treatment, and these treatment can involve medicine, injections, exercises, diet e.t.c. these are to be adhered to, you cannot say I will take medicine today, I will not take tomorrow".

Another social worker said:

"As an out-patient, when one buys drugs, the patient has to buy medicine till the next appointment, because these drugs cannot be sold anywhere else, and these patients come from far and wide".

There is also a loss of family life, as early onset dementia can disrupt family transitional stages, example the children can find it hard to leave home, preventing the family from moving on to the next stage. Also, sufferers are likely to have young children and the children may feel that they are responsible for the condition, due to something that they have thought, said or done, a doctor, who is a key informant, said thus:

\begin{abstract}
"If patients have younger children, they behave in an aggressive way to the parent, who is ill; there is also a loss of personal relationships, which leads to loss of social status, as sexual interest lessens or increases, which do not correspond with partner's needs".
\end{abstract}

\title{
5. 4. Difficulties in social situations
}

All respondents described feelings of discomfort when in public places due to early onset of dementia. It is a visible condition that subjects expressed can evoke stares and questions from strangers. Concerns about what others may think or assume was a cause of embarrassment, self-consciousness, and reduced social activity for some. The lack of understanding from others and false assumptions that they may make was described as very frustrating and an everyday challenge.

One participant who is an out-patient was interviewed with her daughter present; the patients' daughter, as she is her mothers' caregiver, said the following:

"I think what's interesting is it's very negative, in my opinion with my mother, she's very self-conscious about it, and she's become much less active as far as going places. She doesn't go out, because she is so selfconscious about her movement, about the loss of memory. 
And talking on the phone, she's convinced that nobody can understand her and that nobody can read her writing."

Another respondent in her early 50s said:

"But, the illness overall probably keeps me from being able to meet a wide variety of people, and the aggressive behaviour and all that I think do keep people a little bit at bay. I think when you go out, I just think people are maybe a little less likely to approach you. So, that's a strange thing. I think there's a nerd type mentality. When there's a weak one, everybody knows it. She's sick or something's off. That's the weird part of socializing with EOD because it becomes noticeable eventually. If somebody spends at least 20 minutes with you, they notice the hallucinations and they figure it out."

As an administrative officer was kind enough to give me the cost of being an inpatient in the hospital, which is exorbitant and cannot be easily affordable, as there are single rooms, double rooms, special rooms, the male and female wards. The loss of status, the illness brings about is drastic, an out-patient, whom the spouse brought in for appointment, the wife contributed her knowledge to the interview, she said:

"A loss of job leads to a loss of social and financial status, there is a loss of social status, as there is no social life, one is forced to stay at home, even though still active, also friends and relatives may fail to give their support".

\section{5. Restrictions and limitations}

The respondents' spoke of feeling restricted and limited in certain aspects of their lives due to dementia. The physical impact of dementia affected their ability to perform everyday activities and affects their hobbies and careers.

"There have been times when I haven't been able to get food into my mouth on a fork or hold a glass. I couldn't hold a cup probably right now, even though I'm stable, so I avoid that."

A doctor at Federal neuro-psychiatric hospital had this to say about the condition:

"To be honest, there are a lot of days when eating for the patient is so much of a hassle, trying to get the fork or spoon from point $A$ to point $B$ that patients pick and choose. Patients eat things like Pap which is thick, because it will stick to the spoon, so this act makes them lose weight".

Every participant talked about their frustrations with eating, and all but two participants also described writing as a challenge. One participant said that:

"Talking, thinking and writing are hard, at times, even moving." 
Another interviewee described that her writing worsened:

"The condition could get to a point where I was not happy with what I was able to do. My writing became erratic. I could be writing and suddenly it was like my hand was possessed. It goes up and down with these scribbled movements."

Dressing and applying makeup were also frequently brought up as challenges. These tasks involve fine-motor movements with which the participant struggled. A respondent in the study said:

"It was interfering with everyday things...I couldn't put on earrings. You could just forget buttons or snaps. It was getting to the point where it was frustrating".

\section{6. Adaptation, coping and acceptance, loss of status}

Respondents discussed many creative strategies they have developed to manage their condition and adapt to everyday challenges. Coping was assisted by support from family, friends, and support groups, but the role of these support systems was variable. Finally, the positive outlook and attitude about the condition was a very common theme throughout all of the interviews. A social worker said:

"When a patient is writing, there is need to have a nonslip cushion so one can grab onto it, When signing, it is good to have a pen with a good grip on it, not these very thin ballpoint pens that are around in the market."

Several participants had tips to help with eating. Many discussed using a straw to drink beverages and having weighted utensils with thick handles. Several respondents expressed this view:

"I found out that if you have a cup of tea or coffee, you keep the Teaspoon in the cup, because that keeps the tea or coffee from shaking."

The respondents described their experiences with support from both family and friends. As a requirement for study participation, almost all respondents had at least one other family member with early onset of dementia. The respondents were asked about discussions they had had with their families. Four respondents stated that they frequently spoke with other affected family members who were very supportive, because they could understand and relate. One social worker commented:

"I feel like we understand each other. When I speak with the patients who I'm very close with, I'll ask them, "How were you on a scale of 1 to 10 today?" They'll talk about how, you know, it was particularly bad today, or I was hurried today or stressed today or very tired today. The condition was worse. So, I try to pass along some things that I have gotten out of my research in early onset dementia that I think may help." 
As dementia is a condition that takes one out of employment, some of the patients do not have adequate finance, to buy their medication, some of them even resort to begging, with this they have lost their previous status, and the condition can reduce them to individuals of lower status.

Some respondents noted that after their initial diagnosis, they discussed EOD with their family members, but have not discussed it since. Four of the respondents said that this was because their family members were less affected than they, so they did not bring it up. Others did not have a good relationship with their family members and so they did not talk about it again. Four of the respondents said that early onset of dementia (EOD) is "just accepted", within their family, so they do not openly discuss it.

\section{DISCUSSION OF FINDINGS}

In this exploratory study, individuals with early-onset of dementia were interviewed, backed up with key informant interview, had with their doctors and social workers, about the impact this condition has had on their lives. The subtopics identified highlight the key issues and common topics discussed during the interviews. Speaking with these individuals and hearing their stories enabled the capture of an insightful and detailed glimpse into their everyday lives. Through these interviews, it became clear that individuals with early onset of dementia have many challenges-physical, emotional, and even financial and that the impact of this condition on quality of life should not be overlooked.

The incidence of dementia is difficult to ascertain (Ochayi. B, 2006), and an individual getting old is inevitable. The demographic characteristics of Nigerian population have shown that the population is ageing (majority of which are women and rural bearers). The workforce in Nigeria is ageing, that means dementia on the workforce is on the rise. As shown in the study by Louis and Rios (2009), embarrassment is common among individuals with early onset of dementia. Embarrassment and self-consciousness were recurring themes throughout the interviews. Respondents felt anxiety when in public places and around large groups of people or strangers. They did not want their behavior to be noticed, and several respondents said that they could often "feel" people staring at them. The embarrassment experienced by the respondents can reach levels that cause social phobia. Respondents discussed avoiding social situations altogether to reduce distress caused by embarrassment. There appears to be a sense of isolation caused by feeling "different" that can lead to actual isolation, and this may have a significant impact on self-esteem and overall happiness.

In this study, respondents discussed their daily challenges with these, and other, simple tasks. Being able to perform ordinary activities requires extra thought, time, and effort by the respondents. This can not only be frustrating, but can also affect desires and choices. As one interviewee pointed out, she eats foods that are easiest to eat rather than foods she would like to eat. This everyday annoyance can wear on the individuals and greatly affect their behavior. All of the participants had tricks they employed to manage their condition. In order to maintain their abilities, they had to adapt and develop new strategies to perform tasks such as eating and writing. These strategies were helpful for everyday activities as well as in their hobbies and careers. All of the respondents spent time sharing their inventive means of working around their condition. Although tasks were still burdensome, they were able to alleviate some frustration by creating these original tricks. The respondents were able to adapt, and others with Early Onset of Dementia (EOD) would benefit from learning about some of these strategies. Support from both family and friends were also a topic of discussion 
in all of the interviews. Some respondents spoke of early onset of dementia often with their family members because, as individuals associated with them, they could personally understand the burden of the condition. Others would joke about early onset dementia together with their family as a coping mechanism. However, some rarely discussed the condition with family because it was simply an "accepted" part of the family and not a constant cause of concern. Half of the experiences described with friends were supportive. Half of the respondents stated that friends were helpful when their behaviours were especially challenging and that they did not feel the need to hide their behaviour around them. The respondents' comments about their experiences with Early Onset of Dementia (EOD) illustrate the utility of support systems for individuals affected with this condition. In any situation where an individual faces a difficult, having a support system in place or a social worker can be of great benefit. Medical providers should make it a practice to ask about the support systems in their patients' lives. Family and friends can be supportive and necessary for coping. If an individual does not have family or friends to talk to about their difficulties with EOD, a referral to a support group or a social worker would be recommended as a place or person to openly talk, share strategies, and heal.

Also related to social setting, several respondents remarked that they were frustrated with the public's ignorance about EOD and the misconceptions that result. Strangers would often falsely label the subjects as drunk or mad. These assumptions were distressing, and trying to correct these assumptions and educate people about EOD on a daily basis became tiresome for the respondents. Several respondents noted that many people understand or have at least heard of Parkinson's disease, but early onset dementia, a much more common condition causing memory loss, is not well-known. Some respondents discussed the need for increased public awareness. The respondents hoped that greater public knowledge about Early Onset of Dementia (EOD) would make their condition less unusual, and the hurtful comments and assumptions made out of ignorance would be reduced. Healthcare providers should discuss comfortable and concise ways of explaining EOD to strangers. Providers may also give patients educational materials about EOD, which they can hand out to others. Developing a strategy for what to say when false assumptions or questions arise may be helpful in reducing embarrassment and anxiety.

The final key theme identified throughout the interviews was a positive outlook present in all respondents. While a previous study by Louis et al. (2007) has shown a higher rate of depression in individuals with EOD, several respondents felt that there were many worse conditions than early onset of dementia, and said that they felt lucky that this is all they have. They compared early onset of dementia (EOD) to other conditions including Parkinson's disease, and Multiple Sclerosis. Many were hopeful that research would soon identify the cause of EOD and effective treatments would soon follow. Several respondents also expressed that they had accepted EOD and learned to make the best of it.

The researcher is not able to determine from this study, however, whether this positive attitude is present in all individuals with EOD or whether it may be more predominant in the early onset population. Most of the individuals interviewed had developed EOD years or months earlier and, therefore, have had a long time to cope and come to accept the condition. In their minds, they have made peace with their condition although it continues to be a frustration for them. Individuals with a more recent onset of dementia or diagnosis may not have yet developed this positive outlook. Medical providers need to be aware that all individuals with EOD may not have reached a point where they have accepted it, and the coping and psychological state for all patients should be evaluated appropriately. 


\section{CONCLUSIONS}

The study concludes that not nearly enough is known about the unique characteristics of early onset of the disease or the problems faced by those who have them. There also remains an inadequate amount of data available on the actual number of early onset individuals and their condition. This study recommends a number of steps be taken in order to meet the needs of the early onset dementia patients. The conclusions drawn from these new data shed much needed light on the vulnerability and unique circumstances faced by this generation of people, most of whom are baby boomers, who will change the face of disease and health care in coming decades. The analysed data includes extensive personal testimony from people with Alzheimer's, and together with the other reports offers policy makers new insights that may help them address health and long term care needs over the next decade.

\section{Recommendations}

Many steps could be taken to reduce the problems confronted by people with early onset dementia and their families. A first step is greater general awareness of early onset of dementia.

Research to develop more precise information about the number of people with early onset dementia and the diseases and conditions that cause their dementia is essential. This information is needed to support greater general awareness about early onset of dementia, training for medical, residential care and community service providers, and planning for appropriate services for people with the conditions and their families and other care partners. Research on approaches to prevent and delay onset and progression of diseases and conditions that cause early onset dementia is also essential. Government and private organizations that pay for biomedical research should solicit and fund research on early onset dementia, and advocacy for research should include early onset dementia explicitly.

Other steps that could be taken to address the problems include the following:

1. A national education program, with specific and separate components should be aimed at the general public; health care professionals, particularly those in a position to diagnose the disease; family and volunteer caregivers; employers, and caregivers; employers, and human resources personnel.

2. Analysis of work environments for employees who have early onset of dementia with emphasis on workplace accommodations that may enhance their lives and their productivity.

3. Dissemination of information regarding programs that aid people with disabilities, as patients with dementia has a disability.

4. Affordable health insurance for individuals with Early onset of dementia who do not have access to or are not eligible for existing insurance programs.

5. More participation of those with early onset dementia in program planning and public education designed to meet the needs of those affected.

6. Provide training for doctors about how to diagnose early onset of dementia and about the availability of specialized diagnostic centers.

7. Increase awareness of early onset dementia among employers and human resources personnel, including the importance of referrals for disability benefits.

8. Provide information about possible work accommodations for people with early onset dementia and how the legal requirements for work accommodation apply to dementia. 


\section{References}

[1] Ajomale, O (2007) “Ageing in Nigeria- Current State, Social and Economic Implications", African Gerontological society.

[2] Bahro M., Silber E., Sunderland T., Journal of the American Geriatrics Society 43 (1995) 41-46.

[3] Baiyewu O., Jegede R. O., Age and Ageing 21 (1992) 256-261.

[4] Burns A., International Psycho geriatrics 4(1) (1992) 43-54.

[5] Cohen D., The American Journal of Alzheimer's Care and Related Disorders \& Research May/June (1991) 6-11.

[6] Cummings J. L., Benson D. F. (1992). “Dementia: A clinical Approach”, (2nd ed). Boston: Butterworth-Heinemann.

[7] The Guardian Newspaper, October 11, 2011, Article titled, "Nigeria's mental healthcare in distress". Vol. 29, No. 11, 926.

[8] Gilley, D. W. (1993). Behavioural and Affective Disturbances in Alzheimer's Disease. In R. W. Parks, R. F. Zec, \& R. S. Wilson (Eds.), "Neuropsychology of Alzheimer's Disease and other Dementias" (pp. 112-137). New York: Oxford University Press.

[9] Gwyther L., Alzheimer Disease and Associated Disorder 8(3) (1994)110-112.

[10] Harris P. B., Keady J., Alzheimers 'Care Quarterly 5(2) (2004)111-122.

[11] Harrison C., Canadian Journal of Aging 12 (1993) 428-440.

[12] Hausman C. (1992). “Dynamic psychotherapy with elderly demented patients”. In G. M. Jones \& B. L. Miesen (Eds.), Care-giving in dementia: Research and Applications (pp. 181-198). New York: Tavistock/Routledge.

[13] Horgas, A. L., Wahl, H. W., \& Baltes, M. M. (1996). “Dependency in late life”. In L. L. Carstensen, B. A. Edelstein, and L. Dornbrand (Eds.), the practical handbook of clinical gerontology (pp. 54-75). Thousand Oaks CA: SAGE Publications.

[14] Katie Maslow, "Early Onset Dementia: a National Challenge, a Future Crisis". $\mathrm{http} / / \mathrm{www}$.alz.org/Alzheimer association.

Information retrieved on $25^{\text {th }}$ of October, 2011 at $3.05 \mathrm{pm}$.

[15] Kasl-Godley J., Gatz M., Clinical Psychology Review 20(6) (2000) 755-782.

[16] Kester K. O., Adeyeye J. O., Ogunyinka K. O., LASU Journal of Social Sciences 6(1-2) (2007) 146-164.

[17] Kitwood T., Aging and Society 10 (1990) 177-196.

[18] Kitwood T., Bredin K., Aging and Society 12 (1992) 269-287.

[19] Metter E. J., Wilson R. S., (1993). “Vascular Dementias”. In R. W. Parks, R. F. Zec, \& S. Wilson (Eds.), Neuropsychology of Alzheimer's disease and other dementias (pp. 416-437). New York: Oxford University Press.

[20] O’Connor D., International Journal of Aging and Human Development 20(2) (1993) 81-92. 
[21] O'Connor D., Journal of Gerontological Social Work 20(3/4) (1993) 113-128.

[22] Ogunyigbe O. Peter (2004)," Fundamentals of social research", (ed), A. O. Fadeyi and O. A. Adedokun. Landmarks Global-links Concept, Matori, Lagos.

[23] Togonu-Bickersteth F., Nigerian Journal of aging and Social Development 1(1) (20011-1.

[24] Unterbach D., Journal of Gerontological Social Work 22(3/4) (1994) 83-94. 